Article

\title{
Influence of Chain-Extension Reaction on Stereocomplexation, Mechanical Properties and Heat Resistance of Compressed Stereocomplex-Polylactide Bioplastic Films
}

\author{
Yodthong Baimark ${ }^{1, *}$ and Sumet Kittipoom ${ }^{2}$ \\ 1 Biodegradable Polymers Research Unit, Department of Chemistry and Center of Excellence for Innovation \\ in Chemistry, Faculty of Science, Mahasarakham University, Mahasarakham 44150, Thailand \\ 2 Faculty of Science, Mahidol University, 272 Rama VI Road, Ratchathewi, Bangkok 10400, Thailand; \\ sumet.kii@mahidol.ac.th \\ * Correspondence: yodthong.b@msu.ac.th
}

Received: 9 October 2018; Accepted: 31 October 2018; Published: 2 November 2018

\begin{abstract}
Stereocomplex polylactide (scPLA) films were prepared by melt blending of poly(L-lactide) (PLLA) and poly(D-lactide) (PDLA) with and without an epoxy-based chain extender before compression molding. The obtained scPLA films were characterized through differential scanning calorimetry, X-ray diffractometry (XRD), tensile testing and dimensional stability to heat. XRD patterns revealed that all the scPLA films had only stereocomplex crystallites. The obtained results showed that the chain-extension reaction improved mechanical properties of the scPLA films, however, it suppressed stereocomplexation and heat resistance.
\end{abstract}

Keywords: polylactide; stereocomplexation; chain extender; mechanical properties; heat resistance

\section{Introduction}

Poly(L-lactic acid) or poly(L-lactide) (PLLA) is one of the most well-known biodegradable bioplastics, because of its low toxicity, bio-renewability, biocompatibility and good processability [1-4]; however, it has limited application because of its poor heat resistance [5,6]. Stereocomplex polylactides (scPLA) prepared by blending between PLLA and poly(D-lactide) (PDLA) have been widely investigated for use as high-performance bioplastics because the scPLA showed better mechanical properties, heat resistance and hydrolysis resistance compared to PLLA $[7,8]$. Highly heat-resistant scPLAs are required for specific applications, such as heat-treatment food packaging, hot fill packaging and microwave applications.

The scPLA have faster crystallization and higher melting points than PLLA due to the stereocomplex crystallites having stronger intermolecular forces than the homo-crystallites [9-11]. Stereocomplexation enhances the crystallization process of the scPLA, which improves its mechanical properties; for PLLA and PDLA, amorphous regions link among stereocomplex crystallites [12-14]. The heat resistance of scPLA is higher than in PLLA, because of faster crystallization and higher melting temperature of stereocomplex crystallites.

These scPLA films have been widely used in research, and are prepared by solution blending $[7,8,11,15-17]$. However, fabrication of scPLA films by melt processing is interesting, because of its possible use in industrial-scale applications. Specimens of scPLA have been prepared by injection [18] and extrusion [19,20]. For these purposes, PLLA and PDLA were melt blended within a screw barrel. To the best of our knowledge, scPLA films prepared by melt blending before compression molding has been scarcely published [21]. Therefore, stereocomplexation, mechanical 
properties and heat resistance of compressed scPLA films needs to be better understood so as to develop practical applications. Thermal chain-scission of PLLA and PDLA during melt blending and compression molding may reduce mechanical properties of the compressed films. Epoxy-based chain extenders have been used to maintain the molecular weight of the PLLA during the melt process through formation of long-chain branching structures [22-24]. However, chain-extension reaction of compressed scPLA films with various PLLA/PDLA ratios has not been reported so far.

Thus, this research work describes the influences of PLLA/PDLA ratio and chain-extension reaction on stereocomplexation, mechanical properties and heat resistance of compressed scPLA films.

\section{Materials and Methods}

\subsection{Materials}

L-Lactic acid (optical purity $>95 \%$, Purac, Rayong, Thailand) and D-lactic acid (optical purity > 99\%, Haihang Industry Co., Ltd., Jinan, China) were used as monomer precursors for synthesizing L-lactide (LLA) and D-lactide (DLA) monomers, respectively, by polycondensation, followed by thermal depolymerisation. These monomers were purified by re-crystallization four times from ethyl acetate before drying in a vacuum oven at $50{ }^{\circ} \mathrm{C}$ for $24 \mathrm{~h}$. 1-Dodecanol (99\%, Sigma-Aldrich, Buchs SG, Switzerland) was purified by distillation under reduced pressure before use. Stannous octoate (Sn(Oct) $)_{2}, 95 \%$, Sigma-Aldrich, Buchs SG, Switzerland) was used without further purification. Epoxy-based chain extender, Joncry ${ }^{\circledR}$ ADR 4368, in flake form was supplied by BASF, Bangkok, Thailand. All reagents used were analytical grade.

\subsection{Synthesis and Characterization of PLLA and PDLA}

High molecular-weight PLLA and PDLA were synthesized by ring-opening polymerization in bulk from the LLA and DLA, respectively, at $165{ }^{\circ} \mathrm{C}$ for $2.5 \mathrm{~h}$ under a nitrogen atmosphere using $\mathrm{Sn}(\mathrm{Oct})_{2}(0.01 \mathrm{~mol} \%)$ and with 1-dodecanol $(0.14 \mathrm{~mol} \%)$ as the initiating system. The obtained PLLA and PDLA were granulated before drying in a vacuum oven at $110{ }^{\circ} \mathrm{C}$ for $3 \mathrm{~h}$ to remove the un-reacted monomers. The \%conversions of both PLLA and PDLA determined from methine proton peaks of polylactide (5.1-5.3 ppm) and un-reacted lactide (4.9-5.1 ppm) of ${ }^{1} \mathrm{H}-\mathrm{NMR}$ spectra (Bruker Anvance $400{ }^{1} \mathrm{H}$-NMR spectrometer, Bruker, Billerica, MA, USA) were 99\%. PLLA and PDLA were characterizaed by Waters e2695 separations module gel permeation chromatograph (GPC, Waters Corporation, Midford, MA, USA), ADP220 polarimeter (Bellingham and Stanley, Kent, UK) and Pyris Diamond differential scanning calorimeter (DSC, Perkin Elmer, Waltham, MA, USA). The results of PLA characteristics are reported in Table 1.

Table 1. Characteristics of poly(L-lactide) (PLLA) and poly(D-lactide) (PDLA).

\begin{tabular}{|c|c|c|c|c|c|}
\hline PLA & L Enantiomer Content a (\%) & $M_{\mathrm{n}}^{\mathrm{b}}(\mathrm{g} / \mathrm{mol})$ & $\mathrm{DI}^{\mathbf{b}}$ & $T_{\mathrm{g}}{ }^{\mathrm{c}}\left({ }^{\circ} \mathrm{C}\right)$ & $T_{\mathrm{m}}{ }^{\mathrm{c}}\left({ }^{\circ} \mathrm{C}\right)$ \\
\hline PLLA & 96.4 & 85,000 & 2.1 & 54 & 173 \\
\hline PDLA & 3.2 & 90,000 & 2.8 & 59 & 176 \\
\hline
\end{tabular}

\subsection{Preparation of scPLA and Their Compressed Films}

PLLA, PDLA and Joncry ${ }^{\circledR}$ were dried in a vacuum oven at $50{ }^{\circ} \mathrm{C}$ overnight before melt blending using a HAAKE Polylab OS Rheomix batch mixer system (Thermo Fisher Scientific, Waltham, MA, USA) at $200{ }^{\circ} \mathrm{C}$ for $4 \mathrm{~min}$ with a rotor speed of $100 \mathrm{rpm}$. Effects of PLLA/PDLA blend ratios (100/0, $90 / 10,80 / 20,70 / 30$ and $60 / 40(w / w))$ with $(4.0 \mathrm{phr})$ and without Joncryl ${ }^{\circledR}$ on properties of scPLAs 
were investigated. The obtained scPLAs were granulated before drying in a vacuum oven at $50{ }^{\circ} \mathrm{C}$ overnight before compression molding.

The compressed scPLA films were prepared using an Auto $\mathrm{CH}$ Carver laboratory press at $240{ }^{\circ} \mathrm{C}$ without any compression force for $1.0 \mathrm{~min}$ and with a 5 ton compression force for $1.0 \mathrm{~min}$ before quickly cooling to room temperature. The film thicknesses were in range $0.2-0.3 \mathrm{~mm}$. The obtained films were kept at room temperature for $24 \mathrm{~h}$ before characterization.

\subsection{Characterization of Compressed scPLA Films}

The thermal transitions of compressed scPLA films were determined using a Pyris Diamond DSC (Perkin Elmer, Waltham, MA, USA) under a nitrogen atmosphere. For DSC, the thermal history of the samples was removed by melting at $250{ }^{\circ} \mathrm{C}$ for $3 \mathrm{~min}$. Then, the sample was quenched to $0{ }^{\circ} \mathrm{C}$ before heating from 0 to $250{ }^{\circ} \mathrm{C}$ at a rate of $10^{\circ} \mathrm{C} / \mathrm{min}$.

The crystalline structures of compressed scPLA films were investigated using a D8 Advance wide-angle X-ray diffractometer (XRD, Bruker, Billerica, MA, USA) at $25^{\circ} \mathrm{C}$ with $\mathrm{CuK} \alpha$ radiation at $40 \mathrm{kV}$ and $40 \mathrm{~mA}$. For XRD, a scan speed of $3^{\circ} / \mathrm{min}$ was chosen to determine the crystalline structures. The degrees of crystallinity from XRD $\left(X_{\mathrm{c}, \mathrm{XRD}}\right)$ of the scPLA films for homo-crystallites ( $\left.\mathrm{hc}-\mathrm{X}_{\mathrm{c}, \mathrm{XRD}}\right)$ and stereocomplex crystallites (sc- $X_{c, X R D}$ ) were calculated by using Equations (1) and (2), respectively [20]:

$$
\begin{aligned}
& \text { hc- } X_{\mathrm{c}, \mathrm{XRD}}(\%)=\mathrm{S}_{\mathrm{hc}} /\left(\mathrm{S}_{\mathrm{hc}}+\mathrm{S}_{\mathrm{sc}}+\mathrm{S}_{\mathrm{a}}\right) \times 100 \\
& \mathrm{sc}-X_{\mathrm{c}, \mathrm{XRD}}(\%)=\mathrm{S}_{\mathrm{sc}} /\left(\mathrm{S}_{\mathrm{hc}}+\mathrm{S}_{\mathrm{sc}}+\mathrm{S}_{\mathrm{a}}\right) \times 100
\end{aligned}
$$

where $S_{\mathrm{hc}}, S_{\mathrm{sc}}$ and $\mathrm{S}_{\mathrm{a}}$ are the diffraction peak areas of homo-crystallites, stereocomplex crystallites and amorphous regions, respectively.

The tensile properties of compressed scPLA films were measured using a LRX + universal mechanical tester (Lloyd Instruments, West Sussex, UK) at $25{ }^{\circ} \mathrm{C}$ and $65 \%$ relative humidity. The films $(100 \times 10 \mathrm{~mm})$ were tested with a gauge length of $50 \mathrm{~mm}$ and a crosshead speed of $50 \mathrm{~mm} / \mathrm{min}$. The tensile properties were averaged from at least five experiments for each sample.

The dimensional stability to heat of compressed scPLA films was tested at $80^{\circ} \mathrm{C}$ for $30 \mathrm{~s}$ under a $200 \mathrm{~g}$ load. Initial length of film samples was $20 \mathrm{~mm}$. The dimensional stability was calculated by Equation (3):

$$
\text { Dimensional stability }(\%)=[\text { initial length }(\mathrm{mm}) / \text { final length }(\mathrm{mm})] \times 100
$$

\section{Results and Discussion}

Compressed scPLA films with and without chain extension were prepared by melt blending followed with compression molding. The scPLA was chain-extended with Joncryl ${ }^{\circledR}$ at the melt-blending step. The relationship of stereocomplexation, mechanical properties and dimensional stability to heat of the compressed scPLA films were studied using various analytical techniques.

\subsection{Thermal Transition Properties}

The thermal transitions of compressed scPLA films were determined from heating DSC thermograms as shown in Figure 1. The DSC results are summarized in Table 2. The $T_{\mathrm{g}}$ and $T_{\mathrm{cc}}$ of the chain-extended scPLA film series were higher than those of the non-chain-extended scPLA film series. This could be explained by long-chain branching structures of the chain-extended scPLA inhibiting chain mobility for glassy-to-rubbery transition and crystallization.

It is interesting that the $\Delta H_{\mathrm{cc}}$ steadily decreased as the PDLA ratio increased for both the non-chain-extended and chain-extended scPLA film series suggesting that PDLA blending enhanced crystallization of the compressed scPLA films during film cooling. This is due to the crystallization of stereocomplex crystallites being faster than that of the homo-crystallites $[7,11]$. 
The $T_{\mathrm{m}, \mathrm{hc}}$ and $T_{\mathrm{m}, \mathrm{sc}}$ of compressed scPLA films were in the ranges $165-173{ }^{\circ} \mathrm{C}$ and $213-241{ }^{\circ} \mathrm{C}$, respectively. The non-chain-extended 60/40 PLLA/PDLA exhibited the highest $T_{\mathrm{m}, \mathrm{sc}}$. This may be due to the $40 \mathrm{wt} \%$ PDLA inducing the largest stereocomplex-crystallites. The $\Delta H_{\mathrm{m} \text {,hc }}$ steadily decreased and $\Delta H_{\mathrm{m}, \mathrm{sc}}$ significantly increased when the PDLA ratios were increased indicating more stereocomplexation of scPLA film matrix. However, the chain-extended scPLA films had lower $\Delta H_{\mathrm{m}, \mathrm{sc}}$ than the non-chain-extended scPLA films for the same PDLA ratio. This suggests that the chain-extension reaction suppressed stereocomplexation of the PLA matrix. The degrees of crystallinity of both the homo- and stereocomplex crystallites of the compressed scPLA films could not be determined from DSC results because of the $T_{\mathrm{cc}}$ peaks of homo- and stereocomplex crystallizations overlapped each other [25].

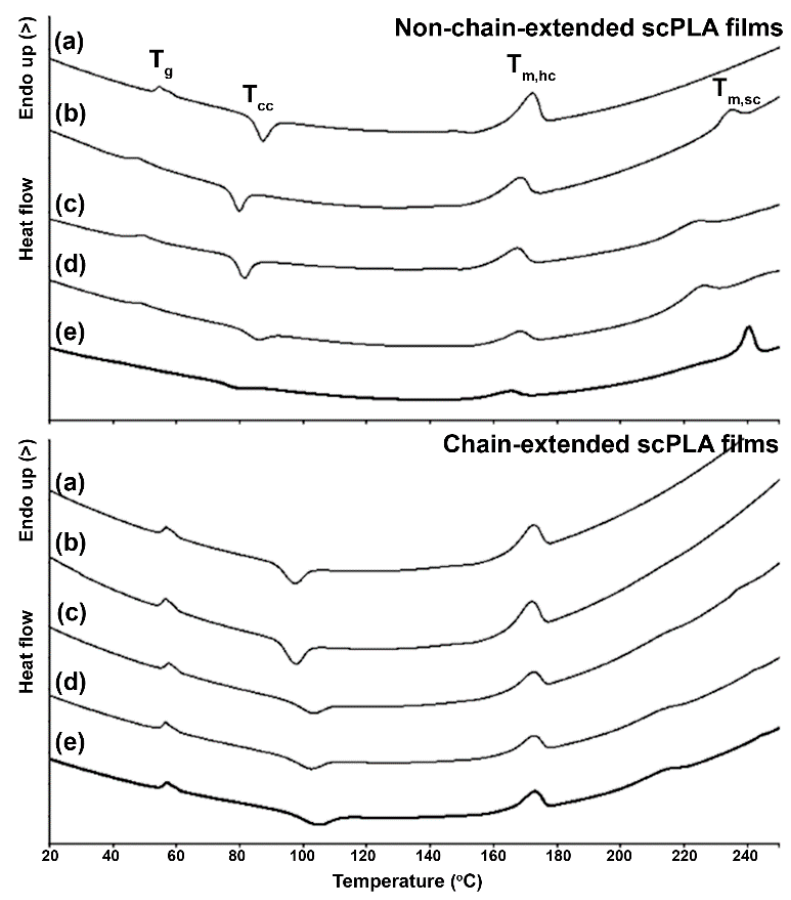

Figure 1. Heating DSC thermograms of (above) non-chain-extended and (below) chain-extended scPLA films with PLLA/PDLA ratios of (a) 100/0, (b) 90/10, (c) 80/20, (d) 70/30 and (e) 60/40 (w/w).

Table 2. Thermal transition properties of stereocomplex polylactide (scPLA) films.

\begin{tabular}{|c|c|c|c|c|c|c|c|}
\hline $\begin{array}{c}\text { PLLA/PDLA Ratio } \\
(w / w)\end{array}$ & $\begin{array}{l}T_{\mathrm{g}} \\
\left({ }^{\circ} \mathrm{C}\right)\end{array}$ & $\begin{array}{l}T_{\mathrm{cc}}{ }^{a} \\
\left({ }^{\circ} \mathrm{C}\right)\end{array}$ & $\begin{array}{c}\Delta H_{\mathrm{cc}}{ }^{\mathrm{a}} \\
(\mathrm{J} / \mathrm{g})\end{array}$ & $T_{\mathrm{m}, \mathrm{hc}}{ }^{\mathrm{a}}$ & $\underset{(\mathrm{J} / \mathrm{g})}{\Delta H_{\mathrm{m}, \mathrm{hc}}{ }^{\mathrm{a}}}$ & $\begin{array}{c}T_{\mathrm{m}, \mathrm{sc}}{ }^{\mathrm{a}} \\
\left({ }^{\circ} \mathrm{C}\right)\end{array}$ & $\begin{array}{c}\Delta H_{\mathrm{m}, \mathrm{sc}}{ }^{\mathrm{a}} \\
\quad(\mathrm{J} / \mathrm{g})\end{array}$ \\
\hline \multicolumn{8}{|l|}{$\begin{array}{l}\text { Non-Chain-Extended } \\
\text { Films }\end{array}$} \\
\hline $100 / 0$ & 52 & 87 & 28.4 & 172 & 52.5 & - & - \\
\hline $90 / 10$ & 44 & 80 & 21.4 & 168 & 38.9 & 234 & 16.3 \\
\hline $80 / 20$ & 44 & 81 & 20.1 & 167 & 32.7 & 223 & 19.6 \\
\hline $70 / 30$ & 45 & 86 & 14.0 & 168 & 19.7 & 225 & 27.0 \\
\hline $60 / 40$ & 41 & 79 & 6.7 & 165 & 11.4 & 241 & 30.6 \\
\hline \multicolumn{8}{|l|}{$\begin{array}{l}\text { Chain-extended } \\
\text { films }\end{array}$} \\
\hline $100 / 0$ & 54 & 97 & 25.9 & 172 & 32.9 & - & - \\
\hline $90 / 10$ & 54 & 98 & 24.2 & 172 & 33.9 & - & - \\
\hline $80 / 20$ & 55 & 103 & 16.4 & 172 & 21.4 & 213 & 2.7 \\
\hline $70 / 30$ & 54 & 102 & 16.2 & 173 & 21.2 & 213 & 6.2 \\
\hline $60 / 40$ & 54 & 105 & 16.3 & 173 & 19.8 & 214 & 9.2 \\
\hline
\end{tabular}

a $T_{\mathrm{cc}}=$ cold crystallization temperature, $\Delta H_{\mathrm{cc}}=$ enthalpy of cold crystallization, $T_{\mathrm{m}, \mathrm{hc}}=$ melting temperature of homo-crystallites, $\Delta H_{\mathrm{m}, \mathrm{hc}}=$ enthalpy of homo-crystallite melting, $T_{\mathrm{m}, \mathrm{sc}}=$ melting temperature of stereocomplex crystallites and $\Delta H_{\mathrm{m}, \mathrm{sc}}=$ enthalpy of stereocomplex-crystallite melting. 


\subsection{Crystalline Structures}

The XRD patterns enabled determination of the crystalline structures of compressed scPLA films as shown in Figure 2. The XRD patterns of both the pure PLLA films with and without chain extension in Figure 2a exhibited a broad underlying 'hump' that was attributed to the XRD patterns for complete amorphous films. The XRD peaks w ere ascribed to crystalline fractions. For scPLA, the XRD peaks of $2 \theta=15^{\circ}, 17^{\circ}$ and $19^{\circ}$ were attributed to homo-crystallites while the XRD peaks of $2 \theta=12^{\circ}, 21^{\circ}$ and $24^{\circ}$ corresponded to stereocomplex crystallites $[26,27]$. It is interesting that all the compressed scPLA films with and without chain extension in Figure $2 \mathrm{~b}-\mathrm{e}$ showed only XRD peaks of stereocomplex crystallites. This may be explained by the compression force under high temperature $\left(T>T_{\mathrm{m}}\right.$,hc $)$ induced stereocomplex formation of the film matrix. It has been reported that external force can enhance sterecomplexation $[15,17,28]$.
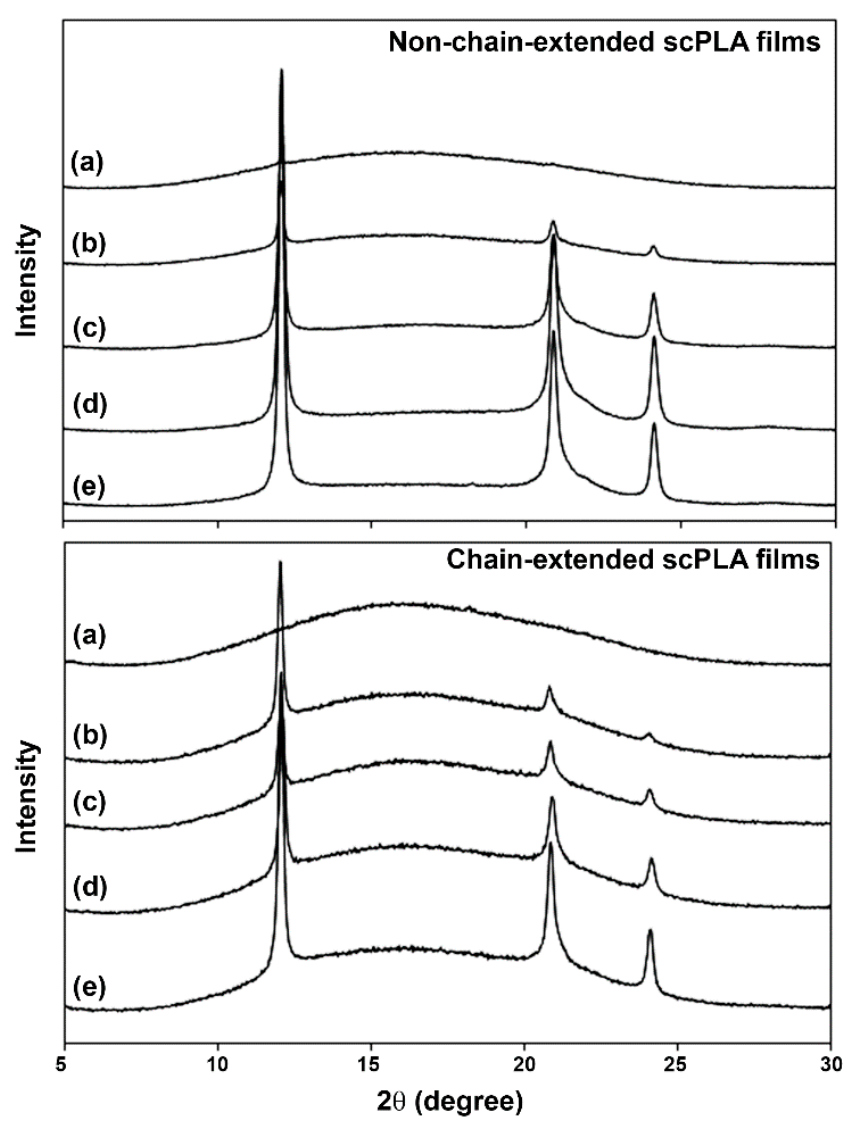

Figure 2. X-ray diffractometry (XRD) patterns of (above) non-chain-extended and (below) chain-extended scPLA films with PLLA/PDLA ratios of (a) 100/0, (b) 90/10, (c) 80/20, (d) 70/30 and (e) 60/40 (w/w).

It can be clearly seen that the intensities of the XRD peaks increased with the PDLA ratios, suggesting an increase of the stereocomplexation of compressed scPLA films. The value for sc- $X_{\mathrm{c}, X \mathrm{XR}}$ can be calculated from the relative areas of the crystalline peaks and the amorphous hump with Equation (2). The values of sc- $X_{c, X R D}$ are compared in Figure 3. The compressed PLLA films with and without chain-extension were completely amorphous as shown in Figure $3 \mathrm{a}\left(\mathrm{sc}-X_{\mathrm{c}, \mathrm{XRD}}=0 \%\right.$ ). The sc- $X_{c, X R D}$ of both the compressed scPLA film series with and without chain-extension in Figure $3 b-e$ increased with the PDLA ratios. The chain-extended scPLA films showed lower sc- $X_{c, X R D}$ than the non-chain-extended scPLA films for the same PDLA ratio. This can be explained by long-chain branching structures of the chain-extended scPLA films that inhibited stereocomplexation according to the DSC results, as described above [29]. It should be noted that the $T_{\mathrm{m}, \mathrm{hc}}$ peaks were detected with the DSC method though not with the XRD method. This may be explained by the homo-crystallization of 
PLA, which could have occurred during the DSC heating scan without compression forces. In addition, PLLA and PDLA homo-crystallization of scPLA films could have not occurred during compression molding at high temperature $\left(T>T_{\mathrm{m}, \mathrm{hc}}\right)$ [20].

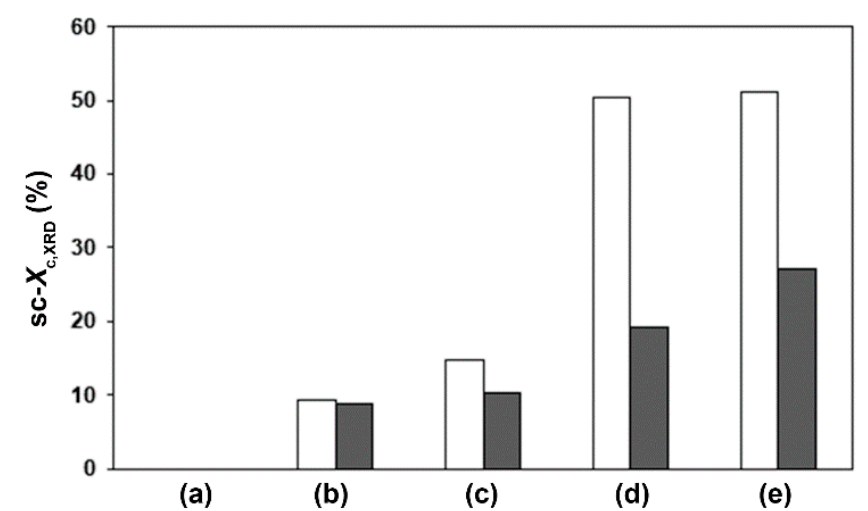

Figure 3. Stereocomplex crystallinity (sc- $X_{c, X R D}$ ) values of $(\square)$ non-chain-extended and ( $\square$ ) chainextended scPLA films with PLLA/PDLA ratios of (a) 100/0, (b) 90/10, (c) 80/20, (d) 70/30 and (e) $60 / 40(w / w)$.

\subsection{Mechanical Properties}

Figure 4 shows the averaged values of tensile properties of the compressed scPLA films, including stress at break, strain at break and Young's modulus. The stress and strain at break of non-chain-extended films with 0-20 wt \% PDLA were in the ranges 41.7-42.3 MPa and 5.2-5.5\%, respectively. It was found that the stress and strain at break of the non-chain-extended $80 / 20$ PLLA/PDLA film in Figure 4c dramatically dropped from 42.7 to $7.5 \mathrm{MPa}$ and 5.3 to $1.7 \%$, respectively, when the PDLA ratio was increased up to $30 \mathrm{wt} \%$ (see Figure $4 \mathrm{~d}$ ). The results indicate that the non-chain-extended 70/30 PLLA/PDLA film was more brittle. This could be explained by the fact that higher sc- $X_{c, X R D}$ values of non-chain-extended scPLA films caused film brittleness (see Figure 3 ). The DSC and XRD results suggested that the stereocomplexation between linear PLLA and PDLA of non-chain-extended scPLA was greater than between long-chain branched PLLA and PDLA of chain-extended scPLA.

However, the chain-extended scPLA film series showed higher stress and strain at break than the non-chain-extended scPLA film series. The results suggested that the chain-extension reaction improved stress and strain at break of compressed scPLA films. From our XRD results, the chain-extension reaction suppressed the sc- $X_{\mathrm{c}, \mathrm{XRD}}$ of scPLA films by maintaining the molecular weights and branching formation of both the PLLA and PDLA. The longer PLLA and PDLA chains in amorphous regions which linked between sterecomplex crystallites could improve tensile properties of the scPLA films [14].

In addition, the stress and strain at the break of the chain-extended scPLA films in Figure $4 \mathrm{~b}-\mathrm{e}$ were higher than both the non-chain-extended and chain-extended PLLA films in Figure 4a. The Young's modulus of non-chain-extended scPLA films decreased as the PDLA ratios increased, whereas the chain-extended scPLA films showed similar Young's modulus in the range 915-1034 MPa. 

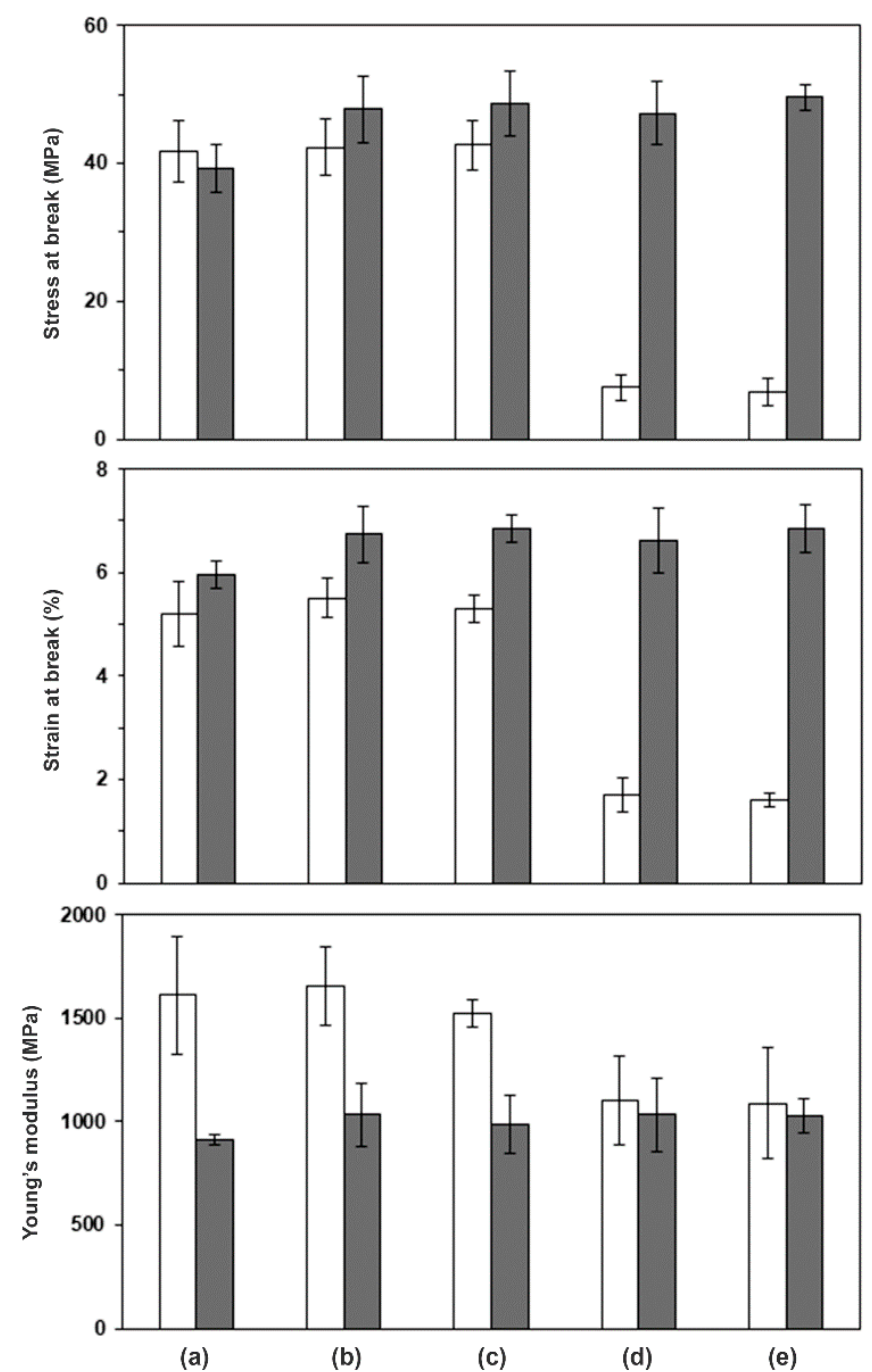

Figure 4. Tensile properties of $(\square)$ non-chain-extended and ( $\square$ ) chain-extended scPLA films with PLLA/PDLA ratios of (a) 100/0, (b) 90/10, (c) 80/20, (d) 70/30 and (e) 60/40 (w/w).

\subsection{Heat Resistance}

The dimensional stability to heat of compressed scPLA films was used to study the heat resistance of film samples. For this purpose, the films were kept at $80{ }^{\circ} \mathrm{C}$ under a $200 \mathrm{~g}$ load for $30 \mathrm{~s} \mathrm{High}$ dimensional-stability of the film samples was attributed to its high heat-resistance. Figure 5 shows film samples before and after dimensional-stability testing. PLLA and 90/10 (w/w) scPLA films in Figure $5 \mathrm{a}, \mathrm{b}$ showed similarly large final lengths for both the non-chain-extended and chain-extended films. The final film lengths significantly decreased as the PDLA ratio increased.

The dimensional-stability values calculated from Equation (3) are clearly compared in Figure 6. The high heat-resistance polypropylene film prepared by the same compression molding had $100 \%$ dimensionally stability (not shown in Figure 6). The PLLA and 90/10 (w/w) scPLA films with and without chain-extension reaction in Figure $6 \mathrm{a}, \mathrm{b}$ had similar dimensional stabilities in range $23.0-26.8 \%$. They had poor heat-resistance because they had low sc- $X_{c, X R D}(0-9.2 \%)$. The dimensional stabilities began increasing when the PDLA ratio was increased up to $20 \mathrm{wt} \%$ (Figure $6 \mathrm{c}$ ). The film of non-chain-extended $60 / 40(w / w)$ scPLA showed the highest dimensional stability $(94.4 \%)$ because it had the largest sc- $X_{\mathrm{c}, X \mathrm{RD}}(51.2 \%)$. The chain-extended scPLA films with PDLA ratios of $20-40 \mathrm{wt} \%$ exhibited lower dimensional-stabilities than the non-chain-extended films for the same PDLA ratio. This can be explained by the lower sc- $X_{\mathrm{c}, X \mathrm{RD}}$ of chain-extended films inducing poor heat-resistance. Therefore, the heat resistance of scPLA strongly depended on its sc- $X_{\mathrm{c}, \mathrm{XRD}}$. 

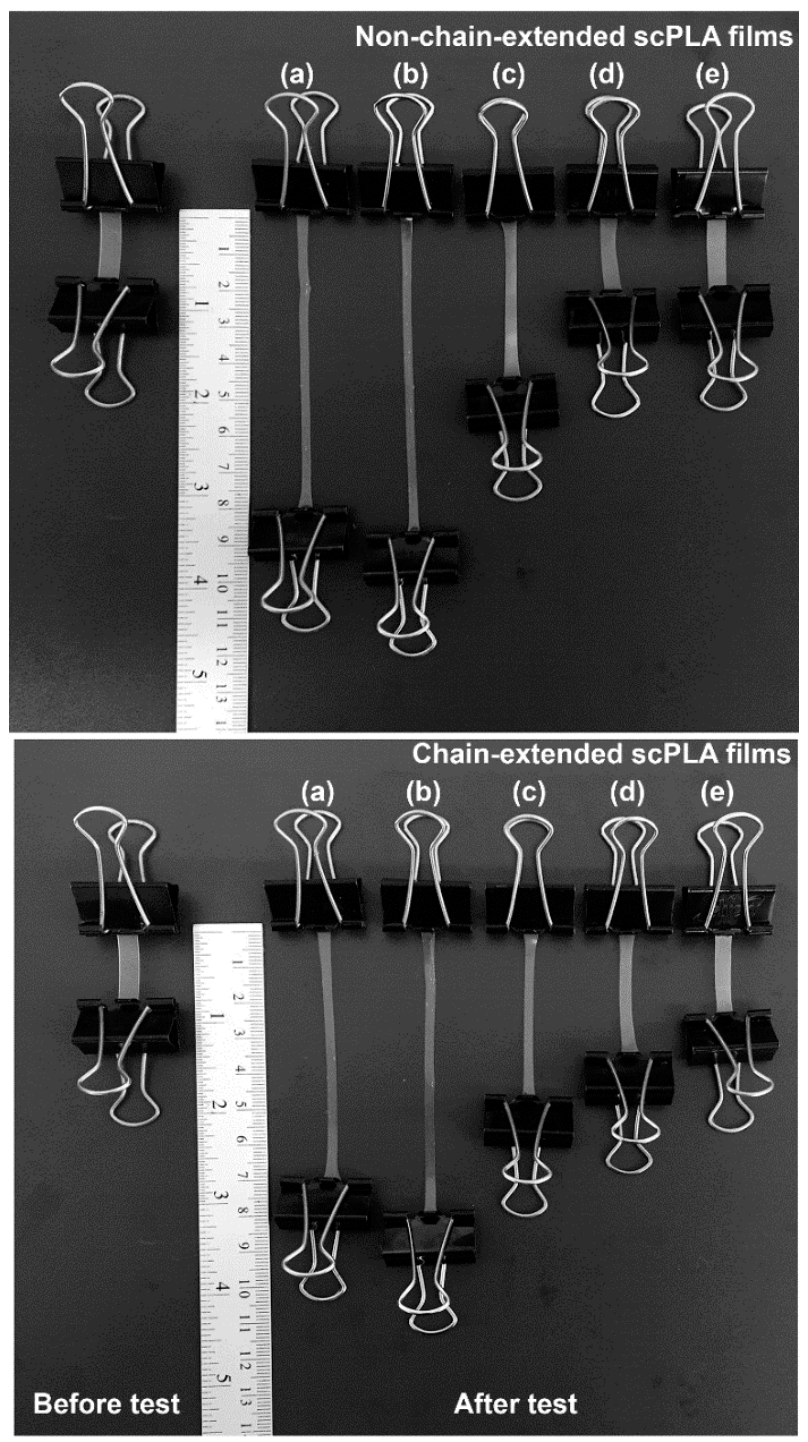

Figure 5. Dimensional stability to heat of (above) non-chain-extended and (below) chain-extended scPLA films with PLLA/PDLA ratios of (a) 100/0, (b) 90/10, (c) 80/20, (d) 70/30 and (e) 60/40 (w/w).

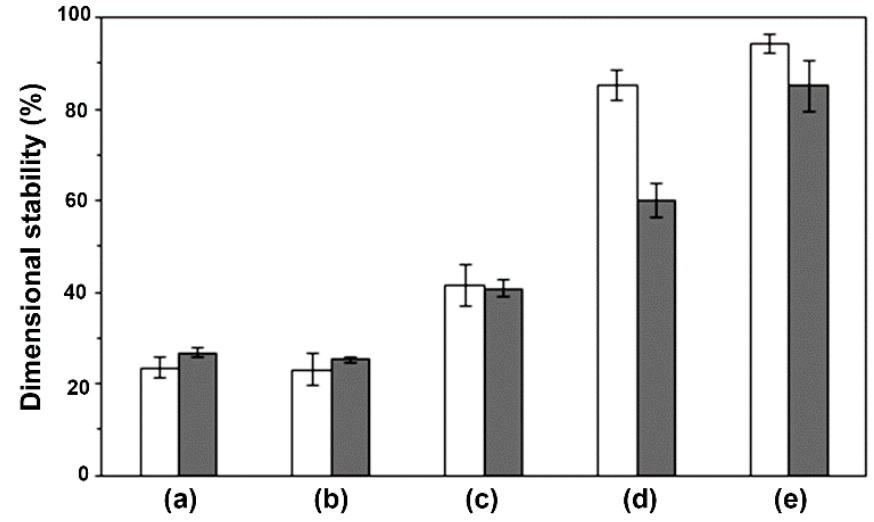

Figure 6. Dimensional stability to heat of $(\square)$ non-chain-extended and ( $\square$ ) chain-extended scPLA films with PLLA/PDLA ratios of (a) 100/0, (b) 90/10, (c) 80/20, (d) 70/30 and (e) 60/40 (w/w). 


\section{Conclusions}

In conclusion, the results reported here show that all the compressed scPLA films exhibited only stereocomplex crystallites as revealed by XRD. The sc- $X_{c, X R D}$ of the compressed scPLA films significantly increased with the PDLA ratio. The chain-extension reaction suppressed stereocomplxation of the scPLA film matrices. However, the non-chain-extended scPLA films exhibited lower stress and strain at break than the chain-extended scPLA films. Therefore, the chain-extension reaction improved tensile properties of the compressed scPLA films. The dimensional stabilities to the heat of compressed scPLA films without chain-extension were as high as $85 \%$ and $94 \%$ for 30 and $40 \mathrm{wt} \%$ PDLA, respectively. The chain-extension reaction reduced dimensional stabilities to the heat of compressed scPLA films containing 30 and $40 \mathrm{wt} \%$ PDLA. The chain-extension reaction decreased both the stereocomplexation and heat resistance of the scPLA films. This work could provide guidance toward fabrication of compressed scPLA products with balanced stereocomplexation, mechanical properties and heat resistance and wider applicability for scPLA.

Author Contributions: Conceptualization, Y.B.; Investigation, S.K.

Acknowledgments: This research was financially supported by the Mahasarakham University (grant no. 6105029). The Center of Excellence for Innovation in Chemistry (PERCH-CIC), Office of the Higher Education Commission, Ministry of Education, Thailand was also acknowledged.

Conflicts of Interest: The authors declare no conflict of interest.

\section{References}

1. Spiridon, L.; Ursu, R.G.; Spiridon, I.A.C. New polylactic acid composites for packaging applications: Mechanical properties, thermal behavior, and antimicrobial activity. Int. J. Polym. Anal. Charact. 2015, 20, 681-692. [CrossRef]

2. Castro-Aguirre, E.; Auras, R.; Selke, S.; Rubino, M.; Marsh, T. Enhancing the biodegradation rate of poly(lactic acid) films and PLA bio-nanocomposites in simulated composting through bioaugmentation. Polym. Degrad. Stab. 2018, 154, 46-54. [CrossRef]

3. Silva, D.; Kaduri, M.; Poley, M.; Adir, O.; Krinsky, N.; Shainsky-Rotiman, J.; Schroeder, A. Biocompatibility, biodegradation and excretion of polylactic acid (PLA) in medical implants and theranostic systems. Chem. Eng. J. 2018, 340, 9-14. [CrossRef]

4. Sangeetha, V.H.; Deka, H.; Varghese, T.O.; Nayak, S.K. State of the art and future prospectives of poly(lactic acid) based blends and composites. Polym. Compos. 2018, 39, 81-101. [CrossRef]

5. Nuzzo, A.; Coiai, S.; Carroccio, S.C.; Dintcheva, N.T.; Gambarotti, C.; Filippone, G. Heat-resistant fully bio-based nanocomposite blends based on poly(lactic acid). Macromol. Mater. Eng. 2014, 299, 31-40. [CrossRef]

6. Zhang, X.; Meng, L.; Li, G.; Liang, N.; Zhang, J.; Zhu, Z.; Wang, R. Effect of nucleating agents on the crystallization behavior and heat resistance of poly(L-lactide). J. Appl. Polym. Sci. 2016, 133, 42999. [CrossRef]

7. Li, Z.; Tan, B.H.; Lin, T.; He, C. Recent advance in stereocomplexation of enantiomeric PLA-based copolymers and applications. Prog. Polym. Sci. 2016, 62, 22-72. [CrossRef]

8. Girdthep, S.; Sankong, W.; Pongmalee, A.; Saelee, T.; Punyodom, W.; Meepowpan, P.; Worajittiphon, P. Enhanced crystallization, thermal properties, and hydrolysis resistance of poly(L-lactic acid) and its stereocomplex by incorporation of graphene nanoplatelets. Polym. Test. 2017, 61, 229-239. [CrossRef]

9. Narita, J.; Katagiri, M.; Tsuji, H. Highly enhanced accelerating effect of melt-recrystallized stereocomplex crystallites on poly(L-lactic acid) crystallization, 2-effects of poly(D-lactic acid) concentration. Macromol. Mater. Eng. 2013, 298, 270-282. [CrossRef]

10. Shao, J.; Xiang, S.; Bian, X.; Sun, J.; Li, G.; Chen, X. Remarkable melting behavior of PLA stereocomplex in linear PLLA/PDLA blends. Ind. Eng. Chem. Res. 2015, 54, 2246-2253. [CrossRef]

11. Shi, X.; Jing, Z.; Zhang, G. Influence of PLA stereocomplex crystals and thermal treatment temperature on the rheology and crystallization behavior of asymmetric poly(L-lactide)/poly(D-lactide) blends. J. Polym. Res. 2018, 25, 71. [CrossRef]

12. Tsuji, H.; Horii, F.; Hyon, S.-H.; Ikada, Y. Stereocomplex formation between enantiomeric poly(lactic acid)s. 2. Stereocomplex formation in concentrated solutions. Macromolecules 1991, 24, 2719-2724. [CrossRef] 
13. Tsuji, H.; Hyon, S.-H.; Ikada, Y. Stereocomplex formation between enantiomeric poly(lactic acid)s. 3. Calorimetric studies on blend films cast from dilute solution. Macromolecules 1991, 24, 5651-5656. [CrossRef]

14. Tsuji, H.; Ikada, Y. Stereocomplex formation between enantiomeric poly(lactic acid)s. XI. mechanical properties and morphology of solution-cast films. Polymer 1999, 40, 6699-6708. [CrossRef]

15. Xu, J.-Z.; Li, Y.; Li, Y.-K.; Chen, Y.-W.; Wang, R.; Liu, G.; Liu, S.-M.; Ni, H.-W.; Li, Z.-M. Shear-induced stereocomplex cylindrites in polylactic acid racemic blends: Morphology control and interfacial performance. Polymer 2018, 140, 179-187. [CrossRef]

16. Lv, T.; Zhou, C.; Li, J.; Huang, S.; Wen, H.; Meng, Y.; Jiang, S. New insight into the mechanism of enhanced crystallization of PLA in PLLA/PDLA mixture. J. Appl. Polym. Sci. 2018, 135, 45663. [CrossRef]

17. Cui, L.; Wang, Y.; Guo, Y.; Liu, Y.; Zhao, J.; Zhang, C.; Zhu, P. Effects of temperature and external force on the stereocomplex crystallization in poly(lactic acid) blends. Adv. Polym. Technol. 2018, 37, 962-967. [CrossRef]

18. Srithep, Y.; Pholharn, D.; Turng, L.-S.; Veang-in, O. Injection molding and characterization of polylactide stereocomplex. Polym. Degrad. Stab. 2015, 120, 290-299. [CrossRef]

19. Pan, G.; Xu, H.; Mu, B.; Yang, J.; Yang, Y. Complete stereo-complexation of enantiomeric for scalable continuous production. Chem. Eng. J. 2017, 328, 759-767. [CrossRef]

20. Pan, G.; Xu, H.; Ma, B.; Wizi, J.; Yang, Y. Polylactide fibers with enhanced hydrolytic and thermal stability via complete stereo-complexation of poly(L-lactide) with high molecular weight of 600000 and lower-molecular-weight poly(D-lactide). J. Mater. Sci. 2018, 53, 5490-5500. [CrossRef]

21. Bai, H.; Deng, S.; Bai, D.; Zhang, Q.; Fu, Q. Recent advances in processing of stereocomplex-type polylactide. Macromol. Rapid Commun. 2017, 38, 1700454. [CrossRef] [PubMed]

22. Jaszkiewicz, A.; Bledzki, A.K.; Meljon, A. Online observations and process analysis of chain extended polylactides during injection moulding. Polym. Degrad. Stab. 2014, 101, 65-70. [CrossRef]

23. Murariu, M.; Paint, Y.; Murariu, O.; Raquez, J.-M.; Bonnaud, L.; Dubois, P. Current progress in the production of PLA-ZnO nanocomposites: Beneficial effects of chain extender addition on key properties. J. Appl. Polym. Sci. 2015, 132, 42480. [CrossRef]

24. Tuna, B.; Ozkoc, G. Effects of diisocyanate and polymeric epoxidized chain extenders on the properties of recycled poly(lactic acid). J. Polym. Environ. 2017, 25, 983-993. [CrossRef]

25. Narita, J.; Katagiri, M.; Tsuji, H. Highly enhanced accelerating effect of melt-recrystallized stereocomplex crystallites on poly(L-lactic acid) crystallization: Effects of molecular weight of poly(D-lactic acid). Polym. Int. 2013, 62, 936-948. [CrossRef]

26. Baimark, Y.; Rungseesantivanon, W.; Prakymoramas, N. Improvement in melt flow property and flexibility of poly(L-lactide)- $b$-poly(ethylene glycol)- $b$-poly(L-lactide) by chain extension reaction for potential use as flexible bioplastics. Mater. Des. 2018, 154, 73-80. [CrossRef]

27. Masutani, K.; Kobayashi, K.; Kimura, Y.; Lee, C.W. Properties of stereo multi-block polylactides obtained by chain-extension of stereo tri-block polylactides consisting of poly(L-lactide) and poly(D-lactide). J. Polym. Res. 2018, 25, 74. [CrossRef]

28. Song, Y.; Zhang, X.; Yin, Y.; de Vos, S.; Wang, R.; Joziasse, C.A.P.; Liu, G.; Wang, D. Enhancement of stereocomplex formation in poly(L-lactide)/poly(D-lactide) mixture by shear. Polymer 2015, 72, 185-192. [CrossRef]

29. Baimark, Y.; Srihanam, P. Influence of chain extender on thermal properties and melt flow index of stereocomplex PLA. Polym. Test. 2015, 45, 52-57. [CrossRef]

(C) 2018 by the authors. Licensee MDPI, Basel, Switzerland. This article is an open access article distributed under the terms and conditions of the Creative Commons Attribution (CC BY) license (http://creativecommons.org/licenses/by/4.0/). 